\title{
Affect and the Computer Game Player: The Effect of Gender, Personality, and Game Reinforcement Structure on Affective Responses to Computer Game-Play
}

\author{
JUSTIN CHUMBLEY, B.Sc. ${ }^{1}$ and MARK GRIFFITHS, Ph.D. ${ }^{2}$
}

\begin{abstract}
Previous research on computer games has tended to concentrate on their more negative effects (e.g., addiction, increased aggression). This study departs from the traditional clinical and social learning explanations for these behavioral phenomena and examines the effect of personality, in-game reinforcement characteristics, gender, and skill on the emotional state of the game-player. Results demonstrated that in-game reinforcement characteristics and skill significantly effect a number of affective measures (most notably excitement and frustration). The implications of the impact of game-play on affect are discussed with reference to the concepts of "addiction" and "aggression."
\end{abstract}

\section{INTRODUCTION}

$\mathbf{E}$ VER SINCE THEIR INTRODUCTION, computer games and their effects have been the subject of widespread speculation. Computer games have been popularly condemned for their encouragement of "addictive" (i.e., recurrent and persistent) game play. This notion has had anecdotal support and great lay appeal. ${ }^{1}$ Early theorists evoked the clinical concept of addiction to explain excessive play. ${ }^{2}$ From this perspective, "computer game addiction" is part of wider behavioral addictions such as pathological gambling and Internet addiction. Griffiths ${ }^{3}$ proposed that computer game addiction may be a function of some internal, personality disposition such as an addictive or dependent personality. In this tradition, attempts have been made to operationalise clinical criteria to assess addictive involvement. 4,5 These scales share criteria with other behavioral addictions (i.e., salience, mood modification, tolerance, withdrawal symptoms, conflict with other activities), and have been used to gather information on addiction prevalence. A number of studies have provided empirical data suggesting that videogame addiction may exist in a small minority of individuals. ${ }^{6}$

A clinical explanation for excessive playing behavior depends on many assumptions. In particular, the extent to which excessive behaviors are qualitatively different from their traditional counterparts. The clinical notion that addictive propensities are discrete and abnormal is contrasted with a growing number of psychological models that postulate a continuum between normal and excessive behaviors. ${ }^{7}$ If behavior does lie on a continuum, studies on "non-clinical" populations may be informative as to the nature of other, more extreme behaviors.

In contrast with clinical attempts to locate the source of excessive game-play in the player, another line of inquiry has looked to in-game stimulus characteristics for the explanation. There is evidence, for example, that people are more willing to play some games than others. In particular, Ma-

${ }^{1}$ Department of Statistical Science, University College, London, United Kingdom.

2International Gaming Research Unit, Nottingham Trent University, Nottingham, United Kingdom. 
lone ${ }^{8}$ found that games that generated high arousal were preferred more. It is clear that computer games can cause physiological arousal..$^{9}$ Furthermore, the available research suggests that this arousal can be manipulated by varying the information rate (i.e., complexity, variability, novelty of stimuli) while permitting users to select desired levels of information rate..$^{10}$ In addition to information rate, it is possible that those characteristics within the game that positively reinforce the player lead to persistent play.11,12 There is evidence, for example, that sound effects and colour displays engender positive affect and increase the games "playability." 8,12 Unfortunately the dearth of hard data on the determinants of persistent play make any strong conclusions on the importance of ingame characteristics impossible.

Attempts to examine the association between computer-games and aggression have also concentrated on in-game factors. In comparison with the scarcity of studies completed on the determinants of persistent play, the subject of aggression has received substantial attention. A number of studies suggest a broad correlation between videogames and aggression. ${ }^{13-15}$ Unfortunately, due to the correlational nature of these studies, neither the direction of causality nor the mechanism is established. It is possible, for example that the correlation between game-play and behavior reflects backwards causation; aggressive people may be attracted to computer games. Furthermore, it is possible that the correlation is explained with reference to some other, extraneous variables.

To examine the issue of causality, some authors have taken to experimental paradigms. Studies amongst child populations have used a physical definition and taken post-game observational measures of behavior. ${ }^{16-18}$ Collectively these studies reinforce that game-play increases the propensity to behaviorally aggress. Given the inappropriateness of broad behavioral measures for adult samples, ${ }^{19}$ the question of which psychological mechanism might explain the propensity of computer games to engender aggression is unanswerable on the basis of any of the aformentioned behavioral data.

It is therefore of importance to our understanding of the relationship between computer games and aggression to determine the importance of affect. In contrast to previous behavioral measures, this requires psychological measurement of affective states. In particular, evidence that game-play increased affective precursors of aggression would support the importance of affective aggression. Due to its high positive correlation with overt ag- gression the sensation of frustration is of particular interest. ${ }^{20}$

In addition to questions concerning the internal constitution of the "aggression," are questions concerning which of the many characteristics of the virtual game environment might influence aggression. The popular cognitive explanation for postplay aggression has emphasised the importance of in-game semantics. In particular, taxonomies of the computer game world have focused on the representation and degree of in-game violence. What such a taxonomy overlooks is that effect of violent content per se may only firmly established if all other qualities of game play are controlled for. There is a possibility, for example, that violence may be confounded with other characteristics of the game. In light of the finding that violent content and graphics are highly related to other important factors such as the rate of in-game action ${ }^{21}$ such a confound may be of significance. It is also possible that factors such as pace, competitiveness and reward/punishment may also confound the distinction between violent and non-violent games. This is important because some of these factors (e.g., competition) may be independent predictors of aggression.

Considering the problems that characterise existing classifications, it is necessary to utilise alternative taxonomies. It appears that a taxonomy based on in-game reinforcement characteristics has both practical and theoretical appeal. By classifying games according to the type, degree, and frequency of reinforcement (positive and negative), one might create a general framework for making comparisons between games that does not depend on their semantic content. Furthermore, because withingame reinforcement can often be varied (by selecting different difficulty levels of the same game), the practical problem of controlling for unnecessary (between-game) variance is bypassed.

Reinforcement is of conceptual interest due to its demonstrated influence on affect and behavior. ${ }^{22-24}$ As such, it provides a framework for the examination of affective aspects of "aggressive" and "addictive" player responses. For example, the frustration-aggression hypothesis ${ }^{23,24}$ states that negative reinforcements can lead to affective aggression if they obstruct with the attainment of an expected and desired goal. Because computer game-play is motivated and intrinsically goalorientated, characteristics associated with success/failure of game objectives might be expected to influence player affect. In-game negative reinforcement should create frustration and increase the propensity to aggress. Conversely, positively 
reinforcing characteristics might be expected to influence the games "playability." Literature on slot machine play indicates that certain reinforcements (e.g., small frequent wins) encourage persistent and/or recurrent play. ${ }^{25}$

The emphasis on in-game reinforcement characteristics does not occlude the influence of other external variables in determining persistent play and post-play aggression. In fact, it is clear from past research that extraneous variables do predict play-related behavior. Most consistently, it appears that males are more likely to play persistently than females. ${ }^{14}$ Unfortunately, in the absence of an explanation it is unclear what other factors internal and external that may underlie this trend. It is possible that factors other than gender differentiate players with different persistence. For example, impulsivity might predict computer game play due to its correlation with boredom. ${ }^{26}$ Furthermore, it might be wise to attempt to delineate the in-game factors that particularly appeal to these different player categories. The fact that reinforcements interact with personality variables to predict behavior ${ }^{27}$ generates hypotheses about the nature of individual differences in-game playing behavior. Impulsives and extraverts, for example, would be expected to be more receptive to positive rather than negative reinforcements. Similarly, differences in skill may underlie differences in player persistence. Because game reinforcement is often contingent of gameplayers skill, it might be expected that skill moderates the influence of game characteristics on affect and behavior.

The concept of reinforcement, therefore, generates a number of hypotheses that ask important questions concerning the alleged propensity for computer games to promote both persistent playing behavior and aggression. Because of the association between reinforcements (offering sensory and psychological rewards and punishments) and affect, this formulation presents an opportunity to test a model that does not rely on the traditional clinical formulations of addiction or social learning theory of aggressive behavior. It was hypothesised that (1) negative reinforcement will be positively related to frustration; (2) positive reinforcement will be positively related to willingness to continue and return to play; (3) impulsive people will be more willing to play and more susceptible to positive reinforcement; and (4) skill should moderate the effect of in-game reinforcement on affect (in particular, skill should be negatively related to frustration).

\section{METHODS}

\section{Participants}

The computer game players were 33 unpaid undergraduates recruited through an advertisement at a Faculty of Cognitive and Computing Science at a Southeastern English university. Their mean age was 21.3 years (range 18-32 years; mode 21 years). Both sexes were represented equally (male $n=17$; female $n=16$ ). Less than half (11 versus 21) of the subjects were frequent players (i.e., played computer games more than one hour per week).

\section{Design}

The experiment had a mixed design. The independent variables were (1) the level of negative reinforcement (within participants); (2) the level of trait impulsivity (between participants); (3) gender; and (4) player experience. The within participants' independent variable was counterbalanced. The dependent variables were (1) self-reported affective responses to the game (including frustration and excitement); (2) self-reported willingness to continue and return to play.

\section{Apparatus and materials}

Software. The game Micromachines operates on a 64-bit Nintendo Playstation computer console. It has a racing format and requires the player to compete with four other (computer-controlled) cars. The aim is to maximise speed and accuracy of the car such that one completes the race before the competitors. Obstacles (e.g., holes, barriers) on the racing track must be avoided as they hinder progress. By altering the difficulty level, the player can simultaneously vary the (a) the proficiency of opponents and (b) the prevalence of obstacles that hinder progress. Controls, game objectives, number of opponents, and general game structure remain constant between difficulty levels. A quicktempoed, repetitive melody accompanied racetime. Acoustic effects reflect success and impediment. Both game conditions were devoid of violent representations.

Controls. The player controls the car by means of a hand held control pad that gives them control over direction (left, right) and speed (accelerate, brake).

Visual display. The games console was attached to a large (24-inch) television monitor. 
Personality measurements. Eysenck's EPS Impulsivity Scale (IVE) ${ }^{27}$ was used as an index of aspects of impulsivity.

"High" and "low" negative reinforcement. Technically, negative reinforcement is the removal of an aversive stimulus that results in increased frequency of a behavior. However, in this study, negative reinforcement is operationally defined as a game condition that is more difficult and involves more challenges leading to more failure experiences. Participants played in one of two conditions where the "high" negative reinforcement condition was the same game but with a higher skill and difficulty level than the "low" negative reinforcement condition.

Affective measurements. A seven-point Likert scale was used to assess eight aspects of participants' post-play mood. Adjectives represented degrees of "negative arousal" (frustration, irritation, boredom, restlessness) and "positive arousal" (excitement, energy, relaxation, calm).

Playability measurements. A seven-point Likert scale was used to present three items measuring participants' propensity to continue and repeat play.

\section{Procedure}

Participants played individually in a soundproofed experimental cubical. Participants were seated in a comfortable chair and invited to fill out an informed consent form and a brief questionnaire about their current game-playing habits and console ownership. Participants were then sat approximately one metre away from the television terminal. They were given verbal information about the game-objective and shown how to control the vehicle using the hand-held control pad. They were then assured that their skill level was not being assessed and that they were not to worry about their game-playing skill or competence. When the experimenter (first author) was content that participants were aware of the instructions and requirements, the first of two versions of the game was loaded. (The order in which each of the participants completed the within-subjects conditions was counterbalanced and participant allocation was randomized.) Players were then instructed to carry on play until such time as the experimenter asked them to stop. The experimenter remained present throughout, sitting to left to the participant facing the opposite direction.
Participants were asked to stop playing after precisely 4 min $40 \mathrm{sec}$. Participants were then required to complete a short questionnaire in which they (a) estimated the time they had been playing, (b) completed items concerning their willingness to continue, and return to, game-play, and (c) indicated how much the game made them feel each of eight affective states. There was also the opportunity to give qualitative comments on what they "liked or disliked" about the experience. The experimenter then loaded the other difficulty condition and participants were required to play for the same period of time. Participants then completed another questionnaire (identical to the first) concerning their perceptions and reactions to the second game. They then completed the IVE scale. After verbal debriefing they were thanked for their participation and invited to ask questions.

\section{Scoring}

Affective measurements. On the basis of the high positive correlations (ranging between $p=0.001$ and $p=0.002$ ) the eight mood categories were collapsed into four dependent variables: negative high-arousal ("frustration"); positive high-arousal ("excitement"); "boredom"; and "calmness."

Playability. Highly significant positive interrelation ( $p=0.001)$ between items inquiring about (a) willingness to continue play, (b) willingness to return to play, and (c) enjoyment, warranted their aggregation into a single measure of "propensity to play."

Impulsivity. Impulsivity scores from the IVE scale were re-coded into three, roughly equal groups representing low (2-5), medium (6-9), and high (10-13) impulsivity.

Player experience. Participants were broadly categorised according to their experience. Participants spending no time playing in the average week were separated from those who played $1 \mathrm{~h}$ or more.

\section{Statistical analysis}

Preliminary analyses found there was no ordering effect on any of the dependent variables. Following confirmation of data homogeneity, a range of mixed design ANOVAs were performed to test for the effects of the independent variables on the dependent variables. 


\section{RESULTS}

\section{Frustration}

Stated frustration ranged from 1 to 7 on a sevenpoint scale. The mean frustration for the low and high negative reinforcement conditions was 3.94 and 4.71 respectively (Table 1 ). A three-way mixed ANOVA (Gender [2] $\times$ Impulsivity [3] $\times$ Reinforcement [2]) showed a main effect of reinforcement on frustration (negative arousal; $p=0.009$ ). An examination of the means showed that the participants in the high negative reinforcement condition experienced more frustration than those in the low negative reinforcement condition. There were no other significant main or interaction effects. Player experience was not included in this analysis due to the relationship between player experience and gender. (Consequentially, cells representing the interaction between Gender, Impulsivity, Reinforcement and Player Experience would have an unacceptably small number of participants.) Consequently, the effect of Player Experience on frustration was examined in a second two-way mixed ANOVA (Player Experience [2] $\times$ Reinforcement [2]). A significant main effect was found for player experience on frustration $(p=0.025)$. Examination of the means shows that experienced players were less frustrated. Similarly there was found to be a significant negative correlation between the number of hours played per week and the degree of frustration $(r=-0.396 ; p=0.012)$.

\section{Excitement}

Stated excitement ranged from 1 to 6.5 on a seven-point scale. Means were 2.92 and 3.56 for the low and high negative reinforcement conditions respectively. Excitement was assessed through a three-way mixed ANOVA (Gender [2] $\times$ Impulsivity [3] $\times$ Reinforcement [2]). Results showed a main effect of reinforcement on excitement $(p=$ 0.023). No other effects were found. An examination of the means showed that the participants in the low negative reinforcement condition were more highly excited than those in the high negative reinforcement condition. A subsequent twoway mixed ANOVA (Player Experience [2] $\times$ Reinforcement [2]) revealed no significant effects of player experience on excitement. However, there was a correlation between excitement and amount of hours played that approached significance $(r=0.287 ; p=0.053)$.

\section{Calmness}

The means for calmness were 2.65 and 2.68 for the high negative and low negative reinforcement conditions respectively (range 1-7). A three-way mixed ANOVA (Gender [2] $\times$ Impulsivity [3] $\times$ Reinforcement [2]) was completed to explain the variance in calmness (positive non-arousal). There were no significant effects or interactions although the effect of gender approached significance $(p=$ 0.064). A subsequent two-way mixed ANOVA (Player Experience [2] $\times$ Reinforcement [2]) revealed no significant effects of player experience on calmness. The correlation between hours played per week and calmness was insignificant.

\section{Boredom}

The means for boredom were 3.00 and 3.94, respectively, for the high negative and low negative reinforcement conditions (range 1-7). A three-way mixed ANOVA (Gender [2] $\times$ Impulsivity [3] $\times$ Reinforcement [2]) found there to be a significant main effect of reinforcement on boredom $(p=$ 0.031). Participants in the low negative reinforcement condition were less bored than those in the high negative reinforcement condition. No other effects were significant. A subsequent two-way mixed ANOVA (Player Experience [2] $\times$ Reinforcement [2]) revealed no significant effect of playerexperience on boredom. The negative correlation between player experience and boredom was nonsignificant for the low reinforcement condition but became significant $(r=-0.309 ; p=0.040)$ for the high reinforcement.

Table 1. Means and Standard Deviations for the Four Affective States for the Both Low Negative/High Positive (L) And High Negative/Low Positive (H)

\begin{tabular}{|c|c|c|c|c|c|c|c|c|}
\hline & \multicolumn{2}{|c|}{ Frustration } & \multicolumn{2}{|c|}{ Excitement } & \multicolumn{2}{|c|}{ Calmness } & \multicolumn{2}{|c|}{ Boredom } \\
\hline & $L$ & $H$ & $L$ & $H$ & $L$ & $H$ & $L$ & $H$ \\
\hline Mean & 3.94 & 4.71 & 3.56 & 2.92 & 2.68 & 2.65 & 3.00 & 3.94 \\
\hline Standard deviation & 1.63 & 1.36 & 1.42 & 1.19 & 1.24 & 1.44 & 1.8 & 1.8 \\
\hline
\end{tabular}




\section{Playability}

Willingness to continue/return to play ranged from 1 to 6 on a seven-point scale. Means were 3.05 and 3.57 for the high negative and low negative reinforcement conditions respectively. A three-way mixed ANOVA (Gender [2] $\times$ Impulsivity [3] $\times$ Reinforcement [2]) confirmed the effect of reinforcement on playability $(p=0.043)$. Participants in the low negative/high positive reinforcement were more willing both to continue and return to gameplay. Neither gender nor impulsivity significantly predicted participants' propensity to play. A further two-way ANOVA (Player Experience [2] $\times$ Reinforcement [2]) showed there to no significant effect of player experience on playability. Within the low reinforcement condition there was a significant positive correlation willingness to play and Excitement (positive arousal; $r=0.559 ; p=0.001$ ). Conversely there was a significant negative correlation between Boredom and willingness to play $(r=$ $-0.643 ; p=0.000)$. Neither Frustration nor Calmness was correlated with willingness to play. The same pattern of significant correlations is observed in the data from the high reinforcement condition. Willingness to play was highly correlated between the two reinforcement conditions (within participants; $r=0.490 ; p=0.002$ ).

\section{Qualitative findings}

Qualitative responses were made on many aspects of game-play and varied between participants. A few people gave positive affective assessments of the game (i.e., they "liked" and "loved" it and found it "fun"). Others displayed negative affective statements and commented that they did not like encountering obstacles and the harshness of the competition. Some also complained about the lack of "intellectual content" and the inability to "explore." Participants generally commented that the controls were tricky to master. Additionally, many participants commented that they were gained some proficiency between the two (within subject) difficulty conditions. Almost invariably, participants found the low negative condition "easier" than the high negative condition.

\section{DISCUSSION}

The results indicate that the reinforcement characteristics within a game can influence the many aspects of the affective state of the player. Of particular interest is the finding that, by increasing the ratio of negative to positive reinforcement, participants experienced more frustration and less excitement. In-game reinforcement characteristics were also found to have an effect on the game's "playability." An increase in the positive reinforcement was associated with a higher propensity to continue and return to play and vice versa. This study also suggests that the player's level of experience was a significant predictor of affective responses to computer game-play. Gender and impulsivity had no effect (direct or indirect) on emotional reactions to game-play. Neither did they effect player's "willingness to continue play." The player's experience, their impulsivity and gender did not predict "propensity to play."

The results of this study indicate that by altering the game's reinforcement characteristics such that they are less rewarding, the degree of frustration experienced increases. This is of interest due to the high positive correlation between frustration and aggression. ${ }^{20}$ Thus, this study constitutes evidence of a possible casual pathway between in-game characteristics and a precursor of affective-aggression. Furthermore, due to the nature of the experimental stimuli, this finding must be explained by factors other than in-game violence and violent representation. It appears that these results fall outside the explanatory scope of traditional formulations, which have emphasised the importance of the cognitive and semantic precursors of aggression. An explanation for why in-game reinforcement ratio influences frustration is found in the frustration-aggression hypothesis.23,24 From this perspective, any negative reinforcements can lead to aggression if they obstruct with the attainment of an expected and desired goal.

What is not clear is whether the frustration response is explained by the meaningfulness of these impediments to the overall goal-acquisition or by their intrinsically "punishing" nature. That is, a question remains over the level at which the computer game per se dictates frustration; directly or as mediated by the players" goals and motivations. While the qualitative responses from the high negative-reinforcement condition bolster the importance of the former (some participants indicated the obstructions and impediments to progress contributed to the sensation of frustration) there is, of course, no reason why these factors are mutually exclusive. It is possible that the influence of one of these factors compounds that of the other. This distinction between levels of influence is of interest because it underlies the implicit question within the research in this area; Is the aggression associated with computerised games peculiar to computer 
games? If the frustration-response is largely explainable on the basis of thwarted goal-acquisition, the affective frustration experienced by computer game players is no different in genesis from that of other games, which involve failure. However, if the frustration-response is intrinsically bound to computerised reinforcement stimuli, then a more negative picture of computer games emerges.

The second hypothesis also received support. In line with Mehrabian and Wixen, ${ }^{11}$ this study supports the idea that in-game factors can determine game persistence. In particular, participants' willingness to play was also effected by the in-game reinforcement characteristics. A higher level of positive reinforcement was associated with an increased propensity to continue and return to play. Furthermore, the affective sensation of excitement was strongly related to this willingness to continue play. As such these results are consistent with the idea elsewhere in the literature that excitement/ arousal underlies game-persistence. An interesting implication of this study is that affect may underlie more extreme forms of playing behavior. This seems feasible if, as with other addictions, one assumes a psychological continuum between normal and excessive behaviors. ${ }^{7}$

Unfortunately, the rather broad manipulation of reinforcement may have confounded several more precise explanations for the observed increase in propensity to play. As before, it is unclear whether it is the informational value concerning goalacquisition that promotes willingness to play or the intrinsically rewarding nature of games' sensory rewards. Without this information, it is unclear of the extent to which compulsive computer gameplay is determined by in-game qualities versus more psychological goals. This critical question concerning the extent to which players become stimulus-bound must be addressed in further research. As before, higher and lower level phenomena are not mutually exclusive and one may compound the effect of the other.

This study also provides some preliminary insights into what deters or terminates play. Interestingly, where a negative correlation between frustration and "willingness to continue play" might be expected, none was found. This finding implies that although high-negative reinforcement engenders frustration, it is not frustration that leads to a termination of play. A concerning implication of this is that player may remain in a frustrating virtual environment for longer than they would in a frustrating environment in the real world. In the absence of any further information one may only speculate why this might be. Unlike the sensation of that of frustration, boredom was found to be negatively related a player's willingness to continue play. It may be that boredom is the crucial mechanism that prompts play-termination. Given the speculative nature of these possibilities it is clear that further research is needed to confirm such speculation.

In contrast to the first two hypotheses, the third was not supported. Players' impulsivity score did not predict their "propensity to play." Prima face, the breadth of the discrepancy between high and low impulsives in this study appears to give this finding credibility. However, this result does certainly not defy the possible importance of personality characteristics on game play. It is possible that the measures used here were too insensitive to detect this effect. Previous evidence suggests that there is generally a low correlation between self-report measures of impulsivity and behavioral ones. ${ }^{28}$ Although the evidence suggests that this correlation becomes positively significant when the behavior is motivated and goal-driven, ${ }^{29}$ it appears that goal-driven behavior in the virtual environment is insufficient. It is possible that another measure of impulsivity would yield positive results.

The fourth hypothesis stated that a player's skill should moderate the effect of game level on frustration. In line with this, it was found that players with more experience had lower feelings of frustration. This may be explained by the fact that the actual ratio of positive to negative reinforcement for each player is contingent on the interaction between that player's skill and the intrinsic structure of the game; better players should therefore receive less negative reinforcement. An implication of this is that factors that correlate with skill might explain demographic trends in the player prevalence. As such, the finding that females are significantly less skillful at computer games ${ }^{30}$ may explain the male bias in the game-playing population. ${ }^{14}$ Unfortunately the results from this study are insufficient to draw definitive conclusions about the importance of skill to frustration. Firstly, the assumption that experience denotes skill may be erroneous; in fact no in-game measures of performance were taken to assess this proposition that more experienced players were actually more skilled. Secondly, there is no direct evidence regarding the causality of the between experience and lower frustration. It is clear that the findings of this study are preliminary and need clarification by way of further inquiry.

More generally, there are three factors that restrict the generalisability of the present findings to other samples and situations. Firstly, the sample consisted of young adults. Consequentially, al- 
though there may be no theoretical reason preventing generalization to children, it is inappropriate to assume a priori results will be found in other age categories. Secondly, due to the solitary playing methodology employed here, it is unclear whether these results apply to games that are played in social context. Finally, it is highly probable that the artificiality of the experimental environment deviated from ethological playing conditions in other ways. For example, in spite of the fact that the experimental preamble assured participants that neither their skill nor their success was being assessed, it is possible participants felt extra pressure to succeed. This goal orientation may have compounded the affective reactions to the game. In addition, there is also a further limitation when considering the external validity of this study. The authors acknowledge that the type of game examined (i.e., a car-racing game) is not as interpersonally violent as other games, therefore conclusions about the effect of the game on violence are limited in this respect.

Despite such limitations, this study has provided some preliminary evidence for the importance of affect to our understanding of computer games. It appears that in-game factors can influence player affect in many ways. This finding is particularly interesting in light of the existent evidence that affect can underlies many aspects of behavior. ${ }^{20}$ An affective approach to computer-game phenomena poses many new questions concerning the aetiology of 'addictive' play and post-play aggression; questions that have, as yet, been occluded by the biases inherent in traditional explanations for these phenomena. Further investigation is needed to examine the precise conditions and degree to which affective responses translate into behaviors. To this end, studies using affective and behavioral measures are particularly pertinent.

\section{REFERENCES}

1. Koop, E. (1982). Surgeon general sees danger in video games. New York Times. 10 Nov:A-16.

2. Soper, W.B., and Miller, M.J. (1986). Junk time Junkies: an emerging addiction among students. School Counsellor 31:40-43.

3. Griffiths, M.D. (1993). Are computer games bad for children? Psychologist: Bulletin of the British Psychological Society 6:401-407.

4. Fisher, S. (1994). Identifying video game addiction in children and adolescents. Addictive Behaviors 19: 545-553.

5. Griffiths, M., and Hunt, N. (1998). Dependence on computer games by adolescents. Psychological Reports 82:475-480.
6. Griffiths, M.D., and Davies, M.N.O. (2005). Videogame addiction: does it exist? In: Goldstein, J., and Raessens, J. (eds.), Handbook of Computer Game Studies. Boston: MIT Press, pp. 359-368.

7. d'Astous, A. (1990). An inquiry into the compulsive side of "normal" consumers. Journal of Consumer Policy 13:51-31.

8. Malone, T.W. (1981). Toward a theory of intrinsically motivating instruction. Cognitive Science 4: 333-369.

9. Griffiths, M.D., and Dancaster, I. (1995). The effect of Type A personality on physiological arousal while playing computer games. Addictive Behaviors 20: 543-548.

10. Mehrabian, H., and Wixen, W.J. (1986). Preference for individual video games as a function of their motional effects on players. Lournal of Applied Social Psychology 16:3-15.

11. Griffiths, M.D. (1997). Computer game playing in early adolescence. Youth and Society 29:223-237.

12. Wood, R.T.A., Griffiths, M.D., Chappell, D., et al. (2004). The structural characteristics of video games: a psycho-structural analysis. CyberPsychology $\mathcal{E} B e-$ havior 7:1-10.

13. Linn, S., and Lepper, M.R. (1987). Correlates of children's usage of video games and computers. Iournal of Applied Social Psychology 17:72-93.

14. Griffiths, M.D., and Hunt, N. (1995). Computer game playing in adolescence: prevalence and demographic indicators. Iournal of Community and Applied Social Psychology 5:189-193.

15. Anderson, C.A., and Bushman, B.J. (2001). Effects of violent video games on aggressive behavior, aggressive cognition, aggressive affect, physiological arousal, and prosocial behavior: a meta-analytic review of the scientific literature. Psychological Science 12:353-359.

16. Silvern, S.B., and Williamson, P.A. (1987). The effects of video game play on young children's aggression, fantasy play and prosocial behaviour. Lournal of Applied and Developmental Psychology 8:453-462.

17. Schutte, N.S, Malouff, J.M, Gorfen, J.C., et al. (1988). The effects of playing games on children's aggressive and other behaviours. Iournal of Applied Social Psychology 18:454-460.

18. Irwin, A.R., \& Gross, A.M. (1995). Cognitive tempo, violent video games, an aggressive behaviour in young boys. Iournal of Family Violence 10:337-350.

19. Winkel, M., Novak, D.M., \& Hopson, H. (1987). Personality factors, subject gender, and the effects of aggressive video games on aggression in adolescents. Journal of Research in Personality 21:211-223.

20. Gustafson, R. (1989). Human physical aggression as a function of magnitude of frustration: Indirect support and a possible confounding influence. Psychological Reports 64:367-374.

21. Anderson, C.A., and Ford, C.M. (1986). Affect of the game player: short-term effects of highly and mildly aggressive video games. Personality and Social Psychology Bulletin 12:390-402. 
22. Witkowski, T., and Stiensmeier Pelster, J. (1998). Performance deficits following failure: learned helplessness or self-esteem protection? British Journal of Social Psychology 37:59-71.

23. Berkowitz, L. (1989). The frustration aggression hypothesis: An examination and reformulation. Psychological Bulletin 106:56-73.

24. Dollard, J., Doob, L.W., Miller, N.E., et al. (1939). Frustration and Aggression. New Haven, CT: Yale University Press.

25. Griffiths, M.D. (1999). The psychology of the near miss (revisited): a comment on Delfabbro and Winefield. British Journal of Psychology 90:441-445.

26. Watt, J., and Vodanovich, J. (1992). A profile of the impulsive personality. Psychological Reports 70:688-690.

27. Eysenck, H.J., and Eysenck, S.B.G. (1996). Manual of the Eysenck Personality Scales (EPS adult) (revised ed.). London: Hodder \& Stoughton Educational.

28. Helmers, K.F., Young, S.N., and Pihl, R.O. (1995). Assessment of measures of impulsivity in healthy male volunteers. Personality and Individual Differences 19: 927-935.

29. Bacharowski, J.A., and Newman, J.P. (1990). Impulsive motor behaviour: effects of personality and goal salience. Iournal of Personality and Social Psychology 58:512-518.

30. Brown, R., Hall, L., Holtzer, R., et al. (1997). Gender and video game performance. Sex-Roles 36:793-812.

Address reprint requests to: Dr. Mark Griffiths

International Gaming Research Unit Psychology Division

Nottingham Trent University Burton Street Nottingham, NG1 4BU, UK

E-mail:mark.griffiths@ntu.ac.uk. 
This article has been cited by:

1. Wei Peng, Ming Liu , Yi Mou . 2008. Do Aggressive People Play Violent Computer Games in a More Aggressive Way? Individual Difference and Idiosyncratic Game-Playing Experience. CyberPsychology \& Behavior 11:2, 157-161. [Abstract] [PDF] [PDF Plus] 